PROCEEDINGS OF THE

AMERICAN MATHEMATICAL SOCIETY

Volume 139, Number 1, January 2011, Pages 177-183

S 0002-9939(2010)10483-5

Article electronically published on July 2, 2010

\title{
DIVISORIAL ZARISKI DECOMPOSITION AND ALGEBRAIC MORSE INEQUALITIES
}

\author{
STEFANO TRAPANI \\ (Communicated by Franc Forstneric)
}

\begin{abstract}
In this paper we use the divisorial Zariski decomposition to give a more precise version of the algebraic Morse inequalities.
\end{abstract}

\section{INTRODUCTION}

In [4 J.-P. Demailly proved the so-called holomorphic Morse inequalities. They relate the cohomology groups of a line bundle on a compact complex manifold to the distribution of the eigenvalues of the curvature of a smooth Hermitian metric on the given line bundle. In [14] we considered the difference $L-F$ of a nef line bundle $L$ and a pseudoeffective line bundle $F$ on a projective manifold in order to derive a more intrinsic version of some of his inequalities. Our statement did not make use of a specific smooth Hermitian metric but it used intersection numbers of the bundles. The same result, in the case where both bundles are nef, was proved and used by Y. T. Siu to derive his effective Matsusaka big theorem [13. A more precise version of this theorem was later obtained in the case of $F$ nef by Demailly in 8 . Even though in 14] we considered the case of $F$ pseudoeffective and not necessarily nef, if $F$ is not nef, a singular Hermitian metric on $F$ comes into the picture, and this makes the results not really intrinsic. The purpose of this paper is to show that the concept of non-nef locus and the divisorial Zariski decomposition obtained in 3. seem to give the correct framework for the algebraic Morse inequalities.

\section{Preliminaries}

Let $X$ be a compact projective manifold of complex dimension $n$. A divisor $F$ on $X$ is said to be pseudoeffective if $c_{1}(F)$ is in the closure in the real Neron-Severi space of the cone of classes of effective $\mathbf{Q}$ divisors. Alternatively, $F$ is pseudoeffective if and only if the line bundle associated to $F$ carries a singular Hermitian metric of non-negative curvature current. More generally a cohomology class in $H^{2}(X, \mathbf{R})$ is said to be pseudoeffective if it is represented by a non-negative closed $(1,1)$ current. Let $T$ be a closed non-negative $(1,1)$-current on $X$, and denote by $\nu_{x}(T)$ the Lelong number of $T$ at $x$. Siu 12 proved that for every positive real number $c$ the set $E_{c}(T)=\left\{x \in X: \nu_{x}(T) \geq c\right\}$ is a proper closed analytic subset of $X$. Let

Received by the editors September 29, 2009 and, in revised form, February 22, 2010

2010 Mathematics Subject Classification. Primary 32L10; Secondary 32J25, 32C30.

Key words and phrases. Divisorial Zariski decomposition, algebraic Morse inequalities, non-nef locus, positive currents. 
$b_{p}(X)=\inf \left\{c>0: \operatorname{codim} E_{c}(T) \geq p\right\}$. For the necessary background on singular metrics, currents, Lelong numbers, currents of minimal singularities and so on, see for example [3, 5, 6, 8,

The stable base locus of $F$ is the intersection of base loci of tensor powers of $F$; that is $\mathbf{B}(F)=\bigcap_{m} B s(m F)$. Let $F$ be a $\mathbf{Q}$ divisor and let $S=\{m \in \mathbf{N}$ : $m F$ is a divisor . The volume of $F$ is by definition given by

$$
\operatorname{Vol}(F)=\limsup _{\substack{m \rightarrow \infty \\ m \in S}} \frac{n ! h^{0}(X, m F)}{m^{n}} .
$$

The volume can be naturally extended to a non-negative continuous function on the set of real pseudoeffective cohomology classes. Recall that the interior of the set of pseudoeffective classes is the set of big classes, i.e., the set of classes represented by a current larger then a Kähler form. Moreover a pseudoeffective class of strictly positive volume is big. Therefore the extension to 0 of the volume function is continuous on all of $H^{2}(X, \mathbf{R})[2$.

Let $A$ be an ample line bundle on $X$. The non-nef locus of $F$ is $E_{n, n}(F)=$ $\bigcup_{k} \mathbf{B}(k F+A)$, and this set in independent of the choice of the ample bundle $A$. Then $F$ is numerically effective (nef) if and only if $E_{n, n}(F)=\emptyset$. Alternatively if $F$ is a pseudoeffective line bundle and $\epsilon$ is a positive rational number, let $T_{\min }(\epsilon)$ be a current of minimal singularity in the family of currents $T$ representing $c_{1}(F)$ such that $T \geq-\epsilon A$. Then $\nu_{x}\left(T_{\min }(\epsilon)\right)$ is decreasing in $\epsilon$. Let $\nu_{x}^{*}(F)=\sup _{\epsilon} \nu_{x}\left(T_{\min }(\epsilon)\right)$. Then $E_{n, n}(F)=\left\{x \in X: \nu_{x}^{*}(F) \neq 0\right\}$; see [3]. A similar notion can be given for the case of pseudoeffective cohomology classes. (The non-nef locus is also called the diminished base locus and is denoted by $\mathbf{B}_{-}(F)$; see for example [9].)

The codimension of $E_{n, n}$ is defined by $\operatorname{codim} E_{n, n}=\min _{k} \operatorname{codim} \mathbf{B}(k F+A)$. So $F$ is nef if and only if $\operatorname{codim} E_{n, n}(F)=n+1$. Since the non-nef locus cannot be of dimension 0 , it follows that $\operatorname{codim} E_{n, n}(F) \neq n$. Moreover for every pseudoeffective divisor $F$ the codimension of $E_{n, n}(F)$ is at least 1 , and it is at least 2 if and only if $F$ is modified nef in the language of [3].

Let $F$ be a pseudoeffective line bundle. In 3 S. Boucksom proves the following facts. There exist a finite number $D_{1}, \ldots, D_{N}$ of codimension-one components of the non-nef locus of $F$. Moreover if we let $\nu_{j}=\min _{x \in D_{j}} \nu_{x}^{*}(F)$, then $\nu_{j}>0$ for $1 \leq j \leq N$ and the non-nef locus of the real divisor $Z(F)=F-\sum_{j} \nu_{j} D_{j}$ has codimension at least 2. See also [11. The decomposition $F=Z(F)+\sum_{j} \nu_{j} D_{j}$ is

called the divisorial Zariski decomposition of $F$. Note that Boucksom's theorem is true in the more general context of real pseudoeffective classes.

Let $T X$ be the tangent bundle of $X$, and let $\pi: P\left(T^{*} X\right)$ be the projectivized cotangent bundle with its natural projection map. Let $\mathbf{O}_{T X}(1)$ be the tautological line bundle over $P\left(T^{*} X\right)$. Let $u$ be a smooth semipositive $(1,1)$-form on $X$. We say that $u$ is a smooth curvature bound if there exists a smooth metric on $\mathbf{O}_{T X}(1)$ with curvature $c\left(\mathbf{O}_{T X}(1)\right)$ such that $c\left(\mathbf{O}_{T X}(1)\right)+\pi^{*}(u) \geq 0$. So for example if the vector bundle $T X$ is ample, we can choose $u=0$.

\section{Algebraic Morse inequalities}

The special case of the theorem below when $F$ is nef was proved in [8, and the proof given here follows similar lines. See also [5].

Theorem 3.1. Let $L$ and $F$ be holomorphic line bundles over $X$, assume that $L$ is nef, that $\operatorname{codim} E_{n, n}(F) \geq q+1$, and let $0 \leq m \leq q$. Then we have the following 
algebraic Morse inequalities:

$$
\begin{aligned}
h^{m}(X, k(L-F)) & \leq \frac{k^{n}}{n !}\left(\begin{array}{c}
n \\
m
\end{array}\right) L^{n-m} F^{m}+o\left(k^{n}\right), \\
(-1)^{m} \sum_{i=0}^{m}(-1)^{i} h^{i}(X, k(L-F)) & \leq(-1)^{m} \frac{k^{n}}{n !} \sum_{i=0}^{m}(-1)^{i}\left(\begin{array}{c}
n \\
i
\end{array}\right) L^{n-i} F^{i}+o\left(k^{n}\right) .
\end{aligned}
$$

Proof. It is sufficient to prove the statement for $m=q$. Let $A$ be an ample divisor, and let $\epsilon$ be a small rational number. By replacing $L$ and $F$ with $L+\epsilon A$ and $F+\epsilon A$ respectively, we may assume that $L$ is ample and $F$ is big. Hence by 3 , Proposition 3.6] $\nu^{*}\left(c_{1}(V), x\right)=\nu\left(T_{\min }, x\right)$, where $T_{\min }$ is a closed positive current with minimal singularities representing $c_{1}(F)$. Choose a positive closed $(1,1)$-form $\omega$ which is the curvature of a smooth metric on the ample line bundle $L$. The assumption that the codimension of $E_{n, n}(F)$ is at least $q+1$ is then the same thing as saying that the superlevel sets $E_{c}\left(T_{\min }\right)$ have codimension larger than or equal to $q+1$ for every positive $c$. Let us choose $u$, a smooth curvature bound. We know from [6] that there exists a sequence of closed smooth forms $T_{k}$ in the cohomology class of $c_{1}(F)$, a decreasing sequence $\lambda_{k}$ of positive continuous functions, and a decreasing sequence of positive real numbers $\epsilon_{k}$ with the following properties:

- the sequence $T_{k}$ converges weakly to $T_{\min }$;

- $\lambda_{k}(x)$ converges to $\nu\left(x, T_{\min }\right)$ for all $x \in X$;

- $\epsilon_{k}$ converges to 0 ;

- $T_{k}>-\lambda_{k} u-2 \epsilon_{k} \omega$.

Let $c$ be a positive real number, and let $\Omega_{k, c}=\left\{x \in X: \lambda_{k}<c\right\}$. Let $v_{k}=$ $2 \epsilon_{k} \omega+c u$, and $w_{k}=\omega+v_{k}$. Then on $\Omega_{k, c}$ the smooth $(1,1)$-forms $T_{k}+v_{k}$ and $w_{k}$ are positive. Now $\omega-T_{k}$ is the curvature of a smooth metric on $L-F$. Let $\alpha_{1} \leq \cdots \leq \alpha_{n}$ be the eigenvalues of $\omega-T_{k}$ with respect to $w_{k}$, so that $\alpha_{j}<1$ for $1 \leq j \leq n$. Let $X(s)=\left\{x \in X: \alpha_{s} \leq 0, \alpha_{s+1} \geq 0\right\}$ and $X(\leq s)=\left\{x \in X: \alpha_{s+1} \geq 0\right\}$. Then on $X(s)$ we have $(-1)^{s}\left(\omega-T_{k}\right)^{n} \leq(-1)^{s} \alpha_{1} \cdots \alpha_{s} w_{k}^{n}$. Now

$$
\left(\begin{array}{c}
n \\
s
\end{array}\right) w_{k}^{n-s} \wedge\left(T_{k}+v_{k}\right)^{s}=\left(\begin{array}{c}
n \\
s
\end{array}\right) w_{k}^{n-s} \wedge\left(w_{k}-\left(\omega-T_{k}\right)\right)^{s}=\sigma_{s}(1-\alpha) w_{k}^{n},
$$

where $\sigma_{s}(1-\alpha)$ is the $s$-th elementary symmetric function in $1-\alpha_{1}, \ldots, 1-\alpha_{n}$. However, since $\alpha_{j}<1$ for $1 \leq j \leq n$, it follows that on $X(s)$ we have

$$
\sigma_{s}(1-\alpha) \geq\left(1-\alpha_{1}\right) \cdots\left(1-\alpha_{s}\right) \geq(-1)^{s} \alpha_{1} \cdots \alpha_{s}
$$

Furthermore one can easily prove by induction on $n[8$ that

$$
\sum_{s=0}^{q}(-1)^{q-s} \sigma_{s}(1-\alpha) \geq \chi_{X(\leq q)}(-1)^{q} \alpha_{1} \cdots \alpha_{n}
$$

where $\chi_{X(\leq q)}$ is the characteristic function of the set $X(\leq q)$. 
Let $\Lambda=\max _{x \in X} \nu\left(T_{\min }, x\right)+1$. From the above we find

$$
\begin{gathered}
\int_{X(q)}(-1)^{q}\left(\omega-T_{k}\right)^{n} \leq\left(\begin{array}{c}
n \\
q
\end{array}\right)\left(\int_{\Omega_{k, c}} w_{k}^{n-q} \wedge\left(T_{k}+v_{k}\right)^{q}\right. \\
+\int_{X \backslash \Omega_{k, c}}\left(\omega+\Lambda u+2 \epsilon_{1} \omega\right)^{n-q} \\
\left.\wedge\left(T_{k}+\Lambda u+2 \epsilon_{1} \omega\right)^{q}\right), \\
\int_{X(\leq q)}(-1)^{q}\left(\omega-T_{k}\right)^{n} \leq \int_{\Omega_{k, c}} \sum_{s=0}^{q}(-1)^{q-s}\left(\begin{array}{c}
n \\
s
\end{array}\right) w_{k}^{n-s} \wedge\left(T_{k}+v_{k}\right)^{s} \\
+\int_{X \backslash \Omega_{k, c}} \sum_{s=0}^{q}(-1)^{q-s}\left(\begin{array}{c}
n \\
s
\end{array}\right)\left(\omega+\Lambda u+2 \epsilon_{1} \omega\right)^{n-s} \\
\wedge\left(T_{k}+\Lambda u+2 \epsilon_{1} \omega\right)^{s} .
\end{gathered}
$$

Consider the form $\Theta_{k, r}=\left(T_{k}+\Lambda u+2 \epsilon_{1} \omega\right)^{r}$ for $0 \leq r \leq q$. Since these forms are in a fixed cohomology class, they have bounded mass; hence up to subsequences they converge as $k \rightarrow \infty$ to an $(r, r)$-current $\Theta_{r}$. Since by assumption if $c>0$, then $\operatorname{codim} E_{c}\left(T_{\min }\right)>q$, it follows that $\Theta_{r}$ has no mass on the sets $E_{c}$.

For $0 \leq s \leq q$ the form $w_{k}^{n-s} \wedge\left(T_{k}+v_{k}\right)^{s}$ is a linear combination with smooth coefficients of the forms $\left(T_{k}+\Lambda u+2 \epsilon_{1} \omega\right)^{r}$ for some $0 \leq r \leq s$. So the forms $w_{k}^{n-s} \wedge\left(T_{k}+v_{k}\right)^{s}$ will also converge to currents with no mass on the sets $E_{c}\left(T_{\min }\right)$. However $\bigcap_{k}\left(X \backslash \Omega_{k, c}\right)=E_{c}\left(T_{\min }\right)$. Therefore

$$
\left|\int_{X} w_{k}^{n-s} \wedge\left(T_{k}+v_{k}\right)^{s}-\int_{\Omega_{k, c}} w_{k}^{n-s} \wedge\left(T_{k}+v_{k}\right)^{s}\right| \rightarrow 0 ;
$$

that is to say, $\lim _{k \rightarrow \infty} \int_{\Omega_{k, c}} w_{k}^{n-s} \wedge\left(T_{k}+v_{k}\right)^{s}=(L+c u)^{n-s}(F+c u)^{s}$. Now we apply the standard holomorphic Morse inequalities, and conclude by letting $c \rightarrow 0$.

An algebraic proof of the above theorem in the case of $F$ nef was given in [1. In the case of $q=1$ and $F$ nef there is a proof by means of the Riemann-Roch theorem, due to Catanese (see [8]).

Let $F$ be a pseudoeffective line bundle; then we have the divisorial Zariski decomposition $F=Z(F)+N(F)$ with $N(F)=\sum_{j=1}^{N} \nu_{j} D_{j}$. Here $\operatorname{codim} E_{n, n}(Z(F)) \geq 2$; hence we obtain the following.

Corollary 3.2. Let $L$ and $F$ be line bundles over $X$, assume that $L$ is nef and $F$ is pseudoeffective, and let $F=Z(F)+N(F)$ be the divisorial Zariski decomposition of $F$. Then

$$
\operatorname{Vol}(L-Z(F)) \geq L^{n}-n L^{n-1} Z(F) \geq L^{n}-n L^{n-1} F .
$$

Proof. Since $L$ is nef, it is sufficient to show that $\operatorname{Vol}(L-Z(F)) \geq L^{n}-n L^{n-1} Z(F)$. If $Z(F)$ is a $\mathbf{Q}$ divisor, the result follows from Theorem [3.1. In the general case, we assume that $L$ is ample and $Z(F)$ is big. Let $\epsilon$ be a small positive number, and let $\nu_{j, k}$ be an increasing sequence of rational numbers converging to $\nu_{j}$. Then $Z_{k}=Z(F)+\sum_{j}\left(\nu_{j}-\nu_{j, k}\right) D_{j}$ is a $\mathbf{Q}$ divisor. Moreover it follows from the fact that the map $N$ is convex and homogeneous [3] that $b_{2}\left(T_{\min }\left(Z_{k}\right)\right)<\epsilon$ for large $k$, where $T_{\min }\left(Z_{k}\right)$ is the current with minimal singularities representing $Z_{k}$. Choose $u$ 
a smooth curvature bound. From the proof of Theorem 3.1 we have $\operatorname{Vol}\left(L-Z_{k}\right) \geq$ $L^{n}-n(L+\epsilon u)^{n-1}\left(Z_{k}+\epsilon u\right)$. Now we let $\epsilon \rightarrow 0$ and $k \rightarrow \infty$.

We observed in 14 that the corollary above is false in general if codim $E_{n, n}(F)=$ 1 and we replaced $Z(F)$ with $F$. However for $q=1$ there is a version of the algebraic Morse inequalities which uses the full divisorial Zariski decomposition.

Theorem 3.3. Let $L$ and $F$ be line bundles over $X$, assume that $L$ is nef and $F$ is pseudoeffective, let $F=Z(F)+N(F)$ be the divisorial Zariski decomposition of $F$ with $N(F)=\sum_{j=1}^{N} \nu_{j} D_{j}$, and let $\{u\}$ be a nef cohomology class in $H^{2}(X, \mathbf{R})$ such that $c_{1}\left(\mathbf{O}_{T X}(1)\right)+\pi^{*}(u)$ is a nef cohomology class in $H^{2}\left(P\left(T^{*} X, \mathbf{R}\right)\right)$. Then

$$
\operatorname{Vol}(L-F) \geq L^{n}-n L^{n-1} Z(F)-n \sum_{j=1}^{N}\left(L+\nu_{j} u\right)^{n-1} \nu_{j} D_{j} .
$$

Proof. As above we see that it is sufficient to prove the theorem for $L$ ample and $F$ big. Furthermore given a positive real number $\epsilon$, by [6, Lemma 6.2] we can find a smooth form $u_{\epsilon}$ in the class $\{u\}$ such that $u_{\epsilon}+\epsilon A$ is a smooth curvature bound. Here $A$ is an ample divisor. If we prove the theorem with $\{u\}$ replaced by $\{u\}+\epsilon A$, then it is sufficient to let $\epsilon \rightarrow 0$ to obtain the general result. So we can further assume that $u$ is a smooth curvature bound. Again, by [3, Proposition 3.6], if $T_{\min }$ is a positive current with minimal singularities representing $F$, then $\nu^{*}\left(c_{1}(F), x\right)=\nu\left(T_{\min }, x\right)$; in particular the divisorial Zariski decomposition $F=Z(F)+\sum_{j=1}^{N} \nu_{j} D_{j}$ is just the Siu decomposition of the current $T_{\min }$ [12. For $1 \leq j \leq N$, let us choose an increasing sequence of rational numbers $\nu_{j, k}$ converging to $\nu_{j}$ as $k \rightarrow \infty$. Let $Z_{k}=Z(F)+\sum_{j}\left(\nu_{j}-\nu_{j, k}\right) D_{j}$ and $N_{k}=\sum_{j} \nu_{j, k} D_{j}$; then $Z_{k}$ and $N_{k}$ are effective $\mathbf{Q}$ divisors such that $F=Z_{k}+N_{k}$. Moreover $\lim _{k \rightarrow \infty} b_{2}\left(T_{\min }-\sum_{j} \nu_{j, k}\left[D_{j}\right]\right)=0$. Let us for the moment fix a positive integer $k$. By [7, proof of Proposition 9.1] there is a sequence $\Delta_{s}$ of effective divisors and a sequence $m_{s}$ of positive integers such that as $s \rightarrow \infty$ we have that:

- the integration currents $\frac{1}{m_{s}}\left[\Delta_{s}\right]$ converge to $T_{\min }-\sum_{j} \nu_{j, k}\left[D_{j}\right]$;

- $b_{2}\left(\frac{1}{m_{s}}\left[\Delta_{s}\right]\right)$ converges to $b_{2}\left(T_{\min }-\sum \nu_{j, k}\left[D_{j}\right]\right)$;

- for $1 \leq j \leq N$ the generic Lelong number $\alpha_{j, s}$ of $\frac{1}{m_{s}}\left[\Delta_{s}\right]$ along $D_{j}$ converges to the generic Lelong number $\nu_{j}-\nu_{j, k}$ of $T_{\min }-\sum_{i} \nu_{i, k}\left[D_{i}\right]$, along $D_{j}$.

So we can write $\frac{1}{m_{s}} \Delta_{s}=\frac{1}{m_{s}} \Delta_{s}^{\prime}+\sum_{j} \alpha_{j, s} D_{j}$, where $\operatorname{codim}\left(\Delta_{s}^{\prime} \cap D_{j}\right) \geq 2$ for $1 \leq j \leq N$. Then $\limsup _{s \rightarrow \infty} b_{2}\left(\frac{1}{m_{s}}\left[\Delta_{s}^{\prime}\right]\right) \leq b_{2}\left(T_{\min }-\sum_{j} \nu_{j, k}\left[D_{j}\right]\right)$. Now we fix $s$ and replace the divisor $F$ by the divisor $F^{\prime}=\frac{1}{m_{s}} \Delta_{s}^{\prime}+\sum_{j}\left(\nu_{j, k}+\alpha_{j, s}\right) D_{j}$. Let $U_{h}(0)$ and $U_{h}(j)$, for $1 \leq j \leq N$, be decreasing fundamental systems of open neighborhoods of $\Delta_{s}^{\prime}$ and $D_{j}$ respectively. Let $\Delta_{0, h}$ and $D_{j, h}$ be sequences of smooth forms approximating for $h \rightarrow \infty$ the currents $\frac{1}{m_{s}}\left[\Delta_{s}^{\prime}\right]$ and $\left(\nu_{j, k}+\alpha_{j, s}\right)\left[D_{j}\right]$ as in the proof of Theorem 3.1 above. By [14, Lemma 2.4] for large $h$ the forms $\Delta_{0, h}$ and $D_{j, h}$ can be taken to have support in $U_{h}(0)$ and in $U_{h}(j)$ respectively. Let $\Theta_{h}=\Delta_{0, h}+\sum_{j} D_{j, h}$; then $\omega-\Theta_{h}$ is the curvature of a smooth metric on $L-F^{\prime}$. Observe that if we look at such a metric for large $h$, then $X_{1} \subseteq U_{h}(0) \cup \bigcup_{j} U_{h}(j)$, and for $K \subseteq X \backslash \Delta_{s}^{\prime} \backslash \bigcup_{j} D_{j}$ compact we may assume that $K \cap\left(U_{h}(0) \cup \bigcup_{j} U_{h}(j)\right)=\emptyset$. Therefore we have $\int_{K}\left(\omega-\Theta_{h}\right)^{n} \geq \int_{K} \omega^{n}$. It follows that

$$
\liminf _{h \rightarrow \infty} \int_{X(0)}\left(\omega-\Theta_{h}\right)^{n} \geq L^{n} \text {. }
$$


Now we use the notation of Theorem 3.1. Let us fix a positive real number $c$ and look at formula (3.1) with $s=1$ on the open set $X_{1} \cap \Omega_{h, c}$. We find that

$$
n w_{h}^{n-1} \wedge\left(\Theta_{h}+v_{h}\right)=\sum_{j=1}^{n}\left(1-\alpha_{j}\right) w_{h}^{n} \geq-\alpha_{1} w_{h}^{n} \geq-\left(\omega-\Theta_{h}\right)^{n} .
$$

Moreover, $w_{h}^{n-1} \wedge\left(\Theta_{h}+v_{h}\right)$ converges weakly to $(\omega+c u)^{n-1} \wedge\left(T_{\min }+c u\right)$ as $h \rightarrow \infty$. For $h$ increasing the measure of $X(1)$ tends to 0 , and therefore

$$
\lim _{h \rightarrow \infty} \int_{X(1)}(\omega+c u)^{n-1} \wedge(c u)=0 .
$$

On the other hand, the intersection of any two distinct divisors $D_{j_{1}}$ and $D_{j_{2}}$ and the intersection of each $D_{j}$ with $\Delta_{s}^{\prime}$ has codimension at least 2. Now for fixed $j$, with $0 \leq j \leq N$, we let $V_{h}(j)=\bigcup_{i \neq j}\left(U_{h}(i) \cap U_{h}(j)\right)$ for $0 \leq i \leq N$. Then as in the proof of Theorem 3.1 we see that

$$
\lim _{h \rightarrow \infty} \int_{X(1) \cap V(j)}-\left(\omega-\Theta_{h}\right)^{n}=0
$$

and that if we take $c_{0}>b_{2}\left(\frac{1}{m_{s}} \Delta_{s}^{\prime}\right)$, then

$$
\begin{aligned}
\limsup _{h \rightarrow \infty} \int_{X(1) \cap U_{h}(0) \backslash V_{h}(0)}-\left(\omega-\Theta_{h}\right)^{n} & \leq n\left(L+c_{0} u\right)^{n-1} \frac{1}{m_{s}}\left[\Delta_{s}^{\prime}\right] \\
& \leq n\left(L+c_{0} u\right)^{n-1} \frac{1}{m_{s}}\left[\Delta_{s}\right] .
\end{aligned}
$$

Similarly if we take $c_{j}>\nu_{j, k}+\alpha_{j, s}$ for $1 \leq j \leq N$, we obtain

$$
\limsup _{h \rightarrow \infty} \int_{X(1) \cap U_{h}(j) \backslash V_{h}(j)}-\left(\omega-\Theta_{h}\right)^{n} \leq n\left(L+c_{j} u\right)^{n-1}\left(\nu_{j, k}+\alpha_{j, s}\right)\left[D_{j}\right] .
$$

Putting everything together and using the standard holomorphic Morse inequalities, we obtain:

$$
\begin{aligned}
& h^{0}\left(X, k\left(L-F^{\prime}\right)\right)-h^{1}\left(X, k\left(L-F^{\prime}\right)\right) \\
& \geq \frac{k^{n}}{n !}\left(L^{n}-n\left(L+c_{0} u\right)^{n-1} \frac{1}{m_{s}}\left[\Delta_{s}\right]\right. \\
& \left.\quad-n \sum_{j=1}^{N}\left(L+c_{j} u\right)^{n-1}\left(\nu_{j, k}+\alpha_{j, s}\right)\left[D_{j}\right]\right)+o\left(k^{n}\right) .
\end{aligned}
$$

We now let $c_{j}$ converge to $\nu_{j, k}+\alpha_{j, s}$ and $c_{0}$ to $b_{2}\left(\frac{1}{m_{s}}\left[\Delta_{s}^{\prime}\right]\right)$. To conclude, first we let $s \rightarrow \infty$, then $k \rightarrow \infty$ and we use the continuity of the volume.

Remark. One can use the results in [10] to improve Corollary 3.2 and Theorem 3.3 by including first cohomology groups as in the standard holomorphic Morse inequalities.

\section{REFERENCES}

[1] F. Angelini, An algebraic version of Demailly's asymptotic Morse inequalities, Proc. Amer. Math. Soc. 124 (1996), no. 11, 3265-3269, DOI 10.1090/S0002-9939-96-03829-4. MR1389502 (97h:32045)

[2] S. Boucksom, On the volume of a line bundle, Internat. J. Math. 13 (2002), no. 10, 1043-1063, DOI 10.1142/S0129167X02001575. MR1945706 (2003j:32025) 
[3] _ Divisorial Zariski decompositions on compact complex manifolds, Ann. Sci. École Norm. Sup. (4) 37 (2004), no. 1, 45-76, DOI 10.1016/j.ansens.2003.04.002 (English, with English and French summaries). MR2050205 (2005i:32018)

[4] J.-P. Demailly, Champs magnétiques et inégalités de Morse pour la d" ${ }^{\prime \prime}$-cohomologie, Ann. Inst. Fourier (Grenoble) 35 (1985), no. 4, 189-229 (French, with English summary). MR812325 (87d:58147)

[5] Singular Hermitian metrics on positive line bundles, Complex algebraic varieties (Bayreuth, 1990), Lecture Notes in Math., vol. 1507, Springer, Berlin, 1992, pp. 87-104, 10.1007/BFb0094512. MR1178721 (93g:32044)

[6] _ Regularization of closed positive currents and intersection theory, J. Algebraic Geom. 1 (1992), no. 3, 361-409. MR1158622 (93e:32015)

[7] _ A numerical criterion for very ample line bundles, J. Differential Geom. 37 (1993), no. 2, 323-374. MR.1205448 (94d:14007)

[8] _ Multiplier ideal sheaves and analytic methods in algebraic geometry, Geometry (Trieste, 2000), ICTP Lect. Notes, vol. 6, Abdus Salam Int. Cent. Theoret. Phys., Trieste, 2001, pp. 1-148. MR1919457 (2003f:32020)

[9] C. D. Hacon and J. McKernan, Existence of minimal models for varieties of log general type. II (August 14, 2008), available at arXiv:0808.1929v1 [math.AG].

[10] A. Küronya, Asymptotic cohomological functions on projective varieties, Amer. J. Math. 128 (2006), 1475-1519. MR2275909 (2007k:14009)

[11] N. Nakayama, Zariski-decomposition and abundance, MSJ Mem., vol. 14, Math. Soc. Japan, Tokyo, 2004. MR2104208(2005h:14015)

[12] Y. T. Siu, Analyticity of sets associated to Lelong numbers and the extension of closed positive currents, Invent. Math. 27 (1974), 53-156. MR0352516 (50:5003)

[13] _ An effective Matsusaka big theorem, Ann. Inst. Fourier (Grenoble) 43 (1993), no. 5, 1387-1405 (English, with English and French summaries). MR 1275204 (95f:32035)

[14] S. Trapani, Numerical criteria for the positivity of the difference of ample divisors, Math. Z. 219 (1995), no. 3, 387-401, DOI 10.1007/BF02572372. MR1339712 (96g:14006)

Dipartimento di Matematica, Università di Roma "Tor Vergata”, Viale della Ricerca SCientifica, 00133 Roma, Italy

E-mail address: trapani@mat.uniroma2.it 